\title{
APPENDIX. GRAIN-SIZE AND CARBON/CARBONATE ANALYSES, LEG 63 ${ }^{1}$
}

\author{
Kenneth A. Pisciotto, Deep Sea Drilling Project, Scripps Institution of Oceanography, La Jolla, California
}

\section{GRAIN-SIZE ANALYSES}

Sand-silt-clay distribution was determined at Scripps on samples collected at the time the cores were split and described. The results are listed in Table 1.

The sediment classification used here is that of Shepard (1954); sand, silt, and clay boundaries are determined on the basis of the Wentworth (1922) scale. Thus the sand, silt, and clay fractions are composed of particles whose diameters range from 2000 to $62.5 \mu \mathrm{m}$, 62.5 to $3.91 \mu \mathrm{m}$, and less than $3.91 \mu \mathrm{m}$, respectively. This classification is applied regardless of sediment type and origin.

Standard sieve and pipette methods were used to determine the grain-size distribution. The sediment sample was dried and dispersed in a Calgon solution. If a sediment sample failed to disaggregate, it was treated with a sonic probe and, if necessary, hydrogen peroxide. Sediment samples that resisted the above treatment were not analyzed.

The sand fraction was removed by wet sieving using a $62.5 \mu \mathrm{m}$ sieve, and the silt and clay fractions were analyzed by standard pipette analysis. Sampling depths and times were calculated using equations derived from the Stokes settling velocity equation (Krumbein and Pettijohn, 1938, pp. 95-96):

$$
\frac{D}{t}=V=\frac{2 g r^{2}\left(d_{1}-d_{2}\right)}{9 \eta}
$$

where $V=$ velocity (in $\mathrm{cm} / \mathrm{s}$ ), $t=$ time (in s), $D=$ depth pipette is inserted (in $\mathrm{cm}$ ), $g=$ gravity (in $\mathrm{cm} / \mathrm{s}$ ), $r$ $=$ radius of individual particles (in $\mathrm{cm}$ ), $d_{1}=$ density of solid particles arbitrarily set at $2.65 \mathrm{~g} / \mathrm{cm}^{3}, d_{2}=$ absolute density of distilled water at different temperatures (Hodgman et al., 1960, p. 2129), and $\eta=$ viscosity of distilled water in poises at different temperatures (Hodgman et al. 1960, p. 2181).

The reproducibility of the grain-size analyses has been previously tested (Boyce, 1972), and it was found that over a period of time with several operators the reproducibility for the sand-silt-clay fractions is $\pm 2.5 \%$ (absolute). For detailed step-by-step procedures, see

\footnotetext{
${ }^{1}$ Initial Reports of the Deep Sea Drilling Project, Volume 63.
}

Volume 4 of the Initial Reports of the Deep Sea Drilling Project.

\section{CARBON/CARBONATE ANALYSES}

Leg 63 sediments were analyzed for total carbon and acid-insoluble (organic) carbon using a LECO WR-12 Analyzer according to the standard technique outlined as follows. The $3-\mathrm{cm}^{3}$ sediment samples were first dried at $105^{\circ} \mathrm{C}$ to $110^{\circ} \mathrm{C}$ and then ground to a homogeneous powder. The ground sediment was redried and two samples, a $0.1-\mathrm{g}$ and a $0.5 \mathrm{-g}$ sample, were then weighed into LECO clay crucibles. The $0.5-\mathrm{g}$ sample was acidified with $10 \%$ hydrochloric acid solution and then washed with distilled water. The sample was then dried and analyzed for acid-insoluble carbon, listed in Table 2 as "organic" carbon. The 0.1-g sample was analyzed for total carbon without further treatment. If the result showed less than $10 \% \mathrm{CaCO}_{3}$, an additional 0.5 -g sample was analyzed for greater accuracy.

The calcium carbonate percentages were calculated as follows:

$$
(\% \text { total } \mathrm{C}-\% \text { organic } \mathrm{C}) \times 8.33=\% \mathrm{CaCO}_{3}
$$

Although other carbonates may be present, all acidsoluble carbon was calculated as calcium carbonate. All results are given in weight percent (Table 2).

Detailed descriptions of the technique and theory may be found in Bader, Gerard, et al. (1970) and Boyce and Bode (1972).

\section{REFERENCES}

Bader, R. G., Gerard, R. D., et al., 1970. Init. Repts. DSDP, 4: Washington (U.S. Govt. Printing Office).

Boyce, R. E., 1972. Grain size analysis, Leg 9, Deep Sea Drilling Project. In Hays, J. D., et al., Init. Repts. DSDP, 9: Washington (U.S. Govt. Printing Office), 779-796.

Boyce, R. E., and Bode, G. W., 1972. Carbon and carbonate analyses, Leg 9, Deep Sea Drilling Project. In Hays, J. D., et al., Init. Repts. DSDP, 9: Washington (U.S. Govt. Printing Office), 797-816.

Hodgman, C. D., Weast, R. C., and Selby, S. M., 1960. Handbook of Chemistry and Physics: Cleveland (Chemical Rubber Publishing Co.).

Krumbein, W. C., and Pettijohn, F. J., 1938. Manual of Sedimentary Petrography: New York (Appleton-Century-Crofts, Inc.).

Shepard, F. P., 1954. Nomenclature based on sand-silt-clay ratios. $J$. Sediment. Petrol., 24:151-158.

Wentworth, C. K., 1922. A scale of grade and class terms for clastic sediments. J. Geol. 30:377-380. 
Table 1. Grain-size analyses, DSDP Leg 63.

\begin{tabular}{|c|c|c|c|c|c|c|c|c|c|}
\hline Sample & $\begin{array}{l}\text { Sub-bottom } \\
\text { Depth } \\
\text { (m) }\end{array}$ & $\begin{array}{c}\text { Sand } \\
(\%)\end{array}$ & $\begin{array}{l}\text { Silt } \\
(\%)\end{array}$ & $\begin{array}{l}\text { Clay } \\
(\%)\end{array}$ & Sample & $\begin{array}{l}\text { Sub-bottom } \\
\text { Depth } \\
\text { (m) }\end{array}$ & $\begin{array}{l}\text { Sand } \\
(\%)\end{array}$ & $\begin{array}{l}\text { Silt } \\
(\%)\end{array}$ & $\begin{array}{l}\text { Clay } \\
(\%)\end{array}$ \\
\hline \multicolumn{5}{|l|}{ Site 467} & \multicolumn{5}{|l|}{ Site 469} \\
\hline $2-3$ & 9.50 & 1.4 & 50.9 & 47.7 & $2-2$ & 9.40 & 0.2 & 45.7 & 54.2 \\
\hline $4-2$ & 27.21 & 1.5 & 40.6 & 57.9 & $3-2$ & 18.88 & 0.7 & 37.4 & 61.9 \\
\hline $6-3$ & 47.67 & 2.8 & 50.2 & 47.0 & $4-2$ & 28.63 & 0.3 & 51.3 & 48.5 \\
\hline $8-5$ & 69.32 & 8.1 & 45.3 & 46.6 & $5-1$ & 37.08 & 0.5 & 44.3 & 55.2 \\
\hline $10-3$ & 85.47 & 2.0 & 37.3 & 60.7 & $6-1$ & 46.62 & 3.0 & 43.0 & 54.0 \\
\hline $12-1$ & 101.42 & 1.9 & 39.7 & 58.4 & $8-2$ & 66.78 & 0.2 & 33.7 & 66.0 \\
\hline $14-3$ & 123.44 & 3.3 & 43.4 & 53.3 & $10-4$ & 89.16 & 0.1 & 25.4 & 74.5 \\
\hline $16-3$ & 142.48 & 1.2 & 55.5 & 43.2 & $13-3$ & 115.60 & 2.7 & 28.1 & 69.1 \\
\hline $18-3$ & 161.31 & 1.2 & 49.2 & 49.6 & $15-2$ & 132.68 & 3.5 & 29.0 & 67.5 \\
\hline $20-2$ & 178.91 & 0.9 & 45.0 & 54.1 & $20-1$ & 179.12 & 0.5 & 17.3 & 82.2 \\
\hline $21-1$ & 187.30 & 2.6 & 49.1 & 48.3 & $23-1$ & 207.80 & 0.5 & 21.2 & 78.3 \\
\hline $25-1$ & 224.82 & 7.2 & 55.5 & 37.3 & $25-1$ & 226.24 & 0.7 & 27.5 & 71.8 \\
\hline $28-1$ & 253.30 & 24.3 & 51.0 & 24.8 & $28-1$ & 254.50 & 9.2 & 52.5 & 38.3 \\
\hline $30-1$ & 272.36 & 8.7 & 58.5 & 32.7 & $31, \mathrm{CC}$ & 283.13 & 1.8 & 42.0 & 56.2 \\
\hline $32-3$ & 294.74 & 12.0 & 58.7 & 29.3 & $33-1$ & 303.29 & 62.9 & 15.3 & 21.8 \\
\hline $34-3$ & 313.62 & 5.0 & 44.4 & 50.6 & \multirow{2}{*}{\multicolumn{5}{|c|}{ Site 470}} \\
\hline $36-2$ & 330.72 & 5.4 & 51.7 & 43.0 & & & & & \\
\hline $42-7$ & 395.35 & 2.4 & 55.2 & 42.4 & $15-1$ & 133.90 & 1.6 & 27.6 & 70.8 \\
\hline $45-4$ & 419.78 & 1.0 & 43.3 & 55.7 & $17-1$ & 153.17 & 0.4 & 25.6 & 74.0 \\
\hline $52-1$ & 481.25 & 0.2 & 49.1 & 50.7 & \multirow{2}{*}{\multicolumn{5}{|c|}{ Site 471}} \\
\hline $54-1$ & 500.76 & 1.2 & 64.9 & 34.0 & & & & & \\
\hline $56-2$ & 521.32 & 1.6 & 76.7 & 21.7 & $1-3$ & 4.02 & 0.1 & 39.3 & 60.6 \\
\hline $59-1$ & 548.87 & 9.1 & 47.9 & 43.0 & $3-2$ & 21.39 & 0.3 & 36.7 & 63.1 \\
\hline $61-1$ & 566.94 & 0.6 & 49.8 & 49.5 & $5-4$ & 43.20 & 0.5 & 33.2 & 66.3 \\
\hline $74-2$ & 692.70 & 5.2 & 67.1 & 27.7 & $7-6$ & 65.59 & 0.2 & 20.5 & 79.4 \\
\hline $81-1$ & 757.36 & 22.4 & 39.5 & 38.1 & $10-3$ & 89.22 & 0.0 & 85.5 & 14.5 \\
\hline $83-1$ & 775.98 & 29.3 & 39.2 & 31.5 & $12-3$ & 108.10 & 0.2 & 21.7 & 78.1 \\
\hline $85-3$ & 798.36 & 12.5 & 46.5 & 41.0 & $15-3$ & 136.70 & 0.2 & 22.0 & 77.8 \\
\hline $96-1$ & 899.68 & 1.7 & 47.8 & 50.5 & $16-2$ & 144.90 & 0.3 & 25.7 & 74.0 \\
\hline $97-1$ & 909.12 & 6.3 & 53.0 & 40.7 & $57-2$ & 534.87 & 7.6 & 52.1 & 40.3 \\
\hline $109-1$ & 1023.17 & 9.4 & 48.5 & 42.1 & $63-3$ & 592.94 & 2.9 & 53.0 & 44.1 \\
\hline \multicolumn{5}{|c|}{ Site 468 , Hole 468} & $65-3$ & 611.69 & 0.5 & 55.7 & 43.8 \\
\hline $1-1$ & 0.70 & 25.7 & 4.2 & 70.0 & \multicolumn{5}{|l|}{ Site 472} \\
\hline $3-1$ & 13.90 & 1.4 & 27.2 & 71.4 & $1-2$ & 2.20 & 0.1 & 31.7 & 68.3 \\
\hline $6-3$ & 45.62 & 7.5 & 38.9 & 53.6 & $2-2$ & 7.10 & 0.0 & 32.1 & 67.9 \\
\hline $7-3$ & 54.40 & 9.9 & 38.9 & 51.2 & $3-1$ & 16.27 & 0.0 & 28.2 & 71.8 \\
\hline $9-4$ & 74.77 & 23.7 & 55.4 & 20.9 & $5-2$ & 36.30 & 0.2 & 21.4 & 78.4 \\
\hline $11-2$ & 91.23 & 79.6 & 15.3 & 5.1 & $7-2$ & 55.20 & 0.8 & 28.5 & 70.7 \\
\hline $18-2$ & 157.46 & 29.9 & 44.5 & 25.6 & $9-1$ & 72.87 & 0.4 & 34.1 & 65.5 \\
\hline \multicolumn{5}{|c|}{ Site 468 , Hole $468 B$} & $11-2$ & 93.36 & 4.0 & 35.4 & 60.6 \\
\hline $2-1$ & 26.25 & 52.9 & 20.8 & 26.3 & \multicolumn{5}{|l|}{ Site 473} \\
\hline $4-1$ & 45.40 & 56.0 & 29.4 & 14.6 & $16-5$ & 139.70 & 0.1 & 38.6 & 61.2 \\
\hline $8-1$ & 83.80 & 2.0 & 36.9 & 61.1 & $18-2$ & 155.30 & 2.7 & 48.9 & 48.4 \\
\hline $9-4$ & 97.56 & 8.4 & 39.8 & 51.8 & $20-3$ & 174.80 & 0.4 & 42.4 & 57.2 \\
\hline $12-1$ & 140.55 & 60.1 & 30.4 & 9.5 & $23-2$ & 192.87 & 0.8 & 37.3 & 62.0 \\
\hline $13-2$ & 161.22 & 8.5 & 55.5 & 36.1 & $25-2$ & 211.09 & 1.4 & 59.8 & 38.8 \\
\hline $15-1$ & 197.21 & 9.8 & 50.2 & 40.0 & $27-1$ & 228.97 & 3.3 & 60.7 & 36.0 \\
\hline $17-1$ & 216.46 & 35.7 & 46.0 & 18.4 & & & & & \\
\hline $31-1$ & 350.27 & 12.3 & 67.2 & 20.5 & & & & & \\
\hline
\end{tabular}


Table 2. Carbon/carbonate analyses, DSDP Leg 63.

\begin{tabular}{|c|c|c|c|c|c|c|c|c|c|c|c|c|c|c|}
\hline $\begin{array}{c}\text { Sample } \\
\text { (interval in } \mathrm{cm} \text { ) }\end{array}$ & $\begin{array}{l}\text { Sub-bottom } \\
\text { Depth } \\
\text { (m) }\end{array}$ & $\begin{array}{l}\text { Total } \\
\text { Carbon } \\
\text { (wt. \%) }\end{array}$ & $\begin{array}{l}\text { Organic } \\
\text { Carbon } \\
\text { (wt. \%) }\end{array}$ & $\begin{array}{l}\mathrm{CaCO}_{3} \\
\text { (wt. \%) }\end{array}$ & $\underset{\text { (interval in } \mathrm{cm} \text { ) }}{\text { Sample }}$ & $\begin{array}{l}\text { Sub-bottom } \\
\text { Depth } \\
\text { (m) }\end{array}$ & $\begin{array}{l}\text { Total } \\
\text { Carbon } \\
\text { (wt. \%) }\end{array}$ & $\begin{array}{l}\text { Organic } \\
\text { Carbon } \\
\text { (wt. \%) }\end{array}$ & $\begin{array}{l}\mathrm{CaCO}_{3} \\
\text { (wt. \%) }\end{array}$ & $\begin{array}{c}\text { Sample } \\
\text { (interval in } \mathrm{cm} \text { ) }\end{array}$ & $\begin{array}{l}\text { Sub-bottom } \\
\text { Depth } \\
\text { (m) }\end{array}$ & $\begin{array}{c}\text { Total } \\
\text { Carbon } \\
\text { (wt. \%) }\end{array}$ & $\begin{array}{l}\text { Organic } \\
\text { Carbon } \\
\text { (wt. \%) }\end{array}$ & $\begin{array}{l}\mathrm{CaCO}_{3} \\
\text { (wt. \%) }\end{array}$ \\
\hline Site 467 & & & & & Site 468 , Hole 468 & & & & & Site 471 & & & & \\
\hline $2-3,45$ & 9.45 & 3.9 & 1.4 & 21 & $1-1,40$ & 0.40 & 9.5 & 0.7 & 74 & $1-3,104$ & 4.04 & 1.7 & 0.7 & 8 \\
\hline $3-3,105$ & 19.55 & 3.1 & 2.0 & 9 & $2-3,100$ & 7.50 & 7.9 & 3.3 & 38 & $3-2,91$ & 21,41 & 1.4 & 0.8 & 6 \\
\hline $4-2,73$ & 27.23 & 4.0 & 1.1 & 24 & $3-1,80$ & 13.80 & 10.2 & 1.1 & 75 & $5-4,67$ & 43.17 & 2.0 & 0.7 & 11 \\
\hline $6-3,69$ & 47.69 & 5.4 & 1.8 & 30 & $5-4,40$ & 36.90 & 8.1 & 1.5 & 55 & $7-6,111$ & 65.61 & 1.1 & 0.9 & 1 \\
\hline $8-5,31$ & 69.31 & 5.1 & 1.9 & 27 & $7-5,40$ & 57.40 & 10.4 & 5.2 & 43 & $10-3,70$ & 89.20 & 1.3 & 1.4 & 0 \\
\hline $8-5,100$ & 70.00 & 6.1 & 1.9 & 35 & $9-4,30$ & 74.80 & 2.2 & 1.0 & 10 & $12-6,60$ & 112.60 & 0.6 & 0.5 & 0 \\
\hline $10-3,48$ & 85.48 & 7.2 & 3.2 & 33 & $11-2,72$ & 91.22 & 0.6 & 0.2 & 3 & $15-2,70$ & 135.20 & 1.1 & 1.1 & 0 \\
\hline $11-2,49$ & 93.49 & 8.3 & 3.1 & 43 & $18-2,45$ & 157.45 & 5.0 & 2.8 & 19 & $16-1,90$ & 143.40 & 0.7 & 0.7 & 0 \\
\hline $12-1,48$ & 101.48 & 8.6 & 2.7 & 49 & $18-3,100$ & 159.50 & 3.5 & 1.5 & 17 & $20-1,10$ & 180.60 & 12.5 & 0.2 & 102 \\
\hline $14-3,47$ & 123.47 & 8.7 & 3.1 & 47 & $21-1,32$ & 184.32 & - & 1.1 & - & $20-1,15$ & 180.65 & - & 0.2 & - \\
\hline $16-3,47$ & 142.47 & 8.0 & 3.5 & 38 & Site 468 Hole 468 A & & & & & $21-1,37$ & 190.37 & - & 0.2 & - \\
\hline $16, \mathrm{CC}, 0$ & 148.52 & - & 1.5 & - & Site 468, Hole 468A & & & & & $22-1,18$ & 199.68 & - & 0.7 & - \\
\hline $17-1,8$ & 148.58 & - & 1.3 & - & $4-5,25$ & 32.25 & 2.2 & 1.4 & 7 & $22-1,38$ & 199.88 & 0.7 & 0.7 & 0 \\
\hline $17-1,8$ & 148.58 & - & 1.1 & - & & & & & & $27, \mathrm{CC}, 18$ & 247.10 & 0.9 & 1.0 & 0 \\
\hline $18-3,30$ & 161.30 & 8.9 & 3.4 & 45 & Site 468 , Hole $468 \mathrm{~B}$ & & & & & $30-1,24$ & 275.74 & 1.5 & 1.4 & 1 \\
\hline $18-5,100$ & 165.00 & 7.6 & 3.6 & 34 & $1-1,40$ & 16.90 & 8.7 & 0.8 & 66 & $31-1,98$ & 285.98 & - & 0.4 & - \\
\hline $20-2,40$ & 178.90 & 6.4 & 3.2 & 27 & $3-1,30$ & 35.80 & 10.8 & 2.0 & 73 & $32-1,19$ & 294.69 & 0.8 & 0.9 & 0 \\
\hline $23-1,0$ & 205.50 & - & 0.8 & - & $6-1,40$ & 64.40 & 10.7 & 1.5 & 77 & $34-2,94$ & 315.94 & 3.4 & 0.7 & 23 \\
\hline $25-1,30$ & 224.80 & 8.0 & 1.7 & 53 & $8-1,40$ & 83.40 & 9.4 & 6.1 & 27 & $36-1,40$ & 332.90 & 1.1 & 1.1 & 0 \\
\hline $26-3,70$ & 237.70 & - & 1.7 & - & $9-4,60$ & 97.60 & 8.5 & 2.6 & 49 & $37, \mathrm{CC}, 15$ & 344.90 & - & 0.1 & - \\
\hline $28-1,77$ & 253.77 & 8.7 & 3.2 & 46 & $12-1,63$ & 140.63 & 8.6 & 2.8 & 49 & $38-2,112$ & 354.12 & 0.7 & 0.7 & 0 \\
\hline $29-1,20$ & 262.70 & 8.5 & 3.4 & 42 & $13-2,71$ & 161.21 & 9.1 & 2.9 & 52 & $39-4,6$ & 365.56 & - & 0.4 & - \\
\hline $30-1,70$ & 272.70 & 4.8 & 1.5 & 27 & $15-1,23$ & 197.23 & 6.6 & 2.8 & 32 & $41-4,28$ & 384.78 & 1.0 & 0.9 & 0 \\
\hline $32-1,63$ & 291.63 & 7.2 & 2.9 & 36 & $17-1,48$ & 216.48 & 2.8 & 2.6 & 1 & $43-2,107$ & 401.57 & 0.4 & 0.5 & 0 \\
\hline $33-3,79$ & 304.29 & - & 1.5 & - & 20, CC, 0 & 244.60 & - & 1.2 & - & $45-1,115$ & 419.15 & 0.4 & 1.1 & 0 \\
\hline $34-3,65$ & 313.65 & 8.9 & 3.3 & 47 & $22-1,30$ & 263.80 & 7.0 & 0.8 & 52 & $47-1,60$ & 437.60 & 1.0 & 0.9 & 1 \\
\hline $36-2,25$ & 330.75 & 5.3 & 2.7 & 21 & $27-1,7$ & 311.07 & - & 0.6 & - & $50-2,65$ & 467.65 & 1.0 & 0.9 & 0 \\
\hline $36-4,7$ & 333.57 & - & 2.1 & - & $28-1,41$ & 320.91 & 4.1 & 1.9 & 18 & $52-2,149$ & 487.49 & 0.7 & 0.6 & 0 \\
\hline $40-1,81$ & 367.81 & - & 1.6 & - & $31-1,125$ & 350.25 & 3.0 & 2.5 & 5 & $57-3,96$ & 535.96 & 0.8 & 0.9 & 0 \\
\hline $40-4,55$ & 372.05 & 8.1 & 2.0 & 51 & Site 469 & & & & & $59-1,71$ & 551.71 & 0.5 & 0.5 & 0 \\
\hline $41-3,33$ & 379.83 & - & 5.3 & - & 3 Ite 409 & & & & & $63-3,97$ & 592.97 & 0.9 & 1.0 & 0 \\
\hline $41-4,100$ & 382.00 & 7.7 & 2.9 & 40 & $1-1,67$ & 0.67 & 1.8 & 0.9 & 7 & $64-3,82$ & 602.32 & - & 0.6 & - \\
\hline $42-5,35$ & 392.35 & 7.4 & 4.0 & 28 & $2-2,30$ & 9.30 & 0.7 & 0.6 & 1 & $65-3,65$ & 611.65 & 0.7 & 0.7 & 0 \\
\hline $45-4,76$ & 419.76 & 5.3 & 4.3 & 8 & $2-2,136$ & 10.36 & 2.9 & 0.5 & 20 & $69-3,94$ & 649.94 & 0.7 & 0.8 & 0 \\
\hline $48-1,57$ & 443.57 & - & 1.1 & - & $3-2,60$ & 19.10 & 1.4 & 1.0 & 3 & $71-2,27$ & 666.77 & 0.5 & 0.6 & 0 \\
\hline $48-2,40$ & 444.90 & 6.3 & 4.6 & 14 & $4-2,60$ & 28.60 & 3.5 & 0.6 & 24 & $73-1,107$ & 685.07 & 0.5 & 0.5 & 0 \\
\hline $52-1,23$ & 481.23 & 6.3 & 3.7 & 22 & $5-1,110$ & 37.10 & 4.0 & 0.6 & 28 & $74-2,68$ & 695.68 & 0.8 & 0.8 & 0 \\
\hline $54-1,72$ & 500.72 & 7.4 & 4.4 & 26 & $6-1,110$ & 46.60 & 6.8 & 0.6 & 52 & $75-3,39$ & 706.39 & 0.6 & 0.6 & 1 \\
\hline $56-2,80$ & 521.30 & 7.2 & 6.0 & 10 & $8-2,75$ & 66.75 & 8.8 & 0.4 & 70 & $76-1,69$ & 713.19 & 0.6 & 0.6 & 1 \\
\hline $57-1,6$ & 528.56 & 7.1 & 4.2 & 24 & $10-4,123$ & 89.23 & 7.4 & 0.5 & 57 & $77-1,133$ & 723.33 & 0.6 & 0.6 & 0 \\
\hline $59-1,128$ & 548.78 & 8.3 & 4.7 & 30 & $12-5,96$ & 109.46 & 3.1 & 0.4 & $\begin{array}{l}23 \\
63\end{array}$ & Site 472 & & & & \\
\hline $\begin{array}{l}61-1,46 \\
64-1,63\end{array}$ & $\begin{array}{l}566.96 \\
595.63\end{array}$ & $\begin{array}{r}12.8 \\
9.1\end{array}$ & $\begin{array}{l}5.6 \\
5.0\end{array}$ & $\begin{array}{r}60 \\
34\end{array}$ & $\begin{array}{l}14-2,60 \\
17-1,34\end{array}$ & $\begin{array}{l}123.60 \\
150.34\end{array}$ & $\begin{array}{l}7.9 \\
8.0\end{array}$ & $\begin{array}{l}0.3 \\
0.1\end{array}$ & $\begin{array}{l}63 \\
66\end{array}$ & $\begin{array}{l}\text { Site } 472 \\
1-1,70\end{array}$ & 0.70 & 0.3 & - & - \\
\hline $66-1,28$ & 614.28 & 5.8 & 4.2 & 14 & $19-1,7$ & 169.07 & 4.0 & 0.4 & 31 & $1-1,70$ & 0.70 & 0.3 & 0.3 & 0 \\
\hline $67-1,35$ & 623.85 & - & 0.5 & - & $22-1,30$ & 197.80 & 7.6 & 0.2 & 62 & $2-1,56$ & 6.06 & 0.2 & 0.2 & 0 \\
\hline $67-1,100$ & 624.50 & - & 1.4 & - & $24-1,110$ & 217.60 & 6.8 & 0.2 & 56 & $3-1,51$ & 15.51 & 0.4 & 0.2 & 1 \\
\hline $68-2,47$ & 634.97 & 8.1 & 3.6 & 38 & $28-1,44$ & 254.94 & 1.7 & 0.7 & 9 & $5-1,80$ & 34.80 & 0.2 & 0.2 & 0 \\
\hline $69-2,15$ & 644.15 & - & 2.7 & - & $31, \mathrm{CC}, 5$ & 283.13 & 0.5 & 0.3 & 1 & $7-1,37$ & 53.37 & 0.2 & 0.2 & 0 \\
\hline $73-1,77$ & 681.27 & 11.8 & 2.6 & 76 & $33-1,129$ & 303.29 & 0.3 & 0.1 & 2 & $8-3,80$ & 66.30 & 0.2 & 0.2 & 0 \\
\hline $76-1,73$ & 709.73 & 7.9 & 1.6 & 52 & $35-1,34$ & 321.34 & 1.0 & 0.2 & 6 & $9-1,54$ & 72.54 & 0.2 & 0.2 & 0 \\
\hline $77-2,10$ & 720.10 & - & 1.3 & - & $37-1,63$ & 340.63 & 0.3 & 0.1 & 2 & $11-2,94$ & 93.44 & 3.2 & 0.2 & 25 \\
\hline $81-1,64$ & 757.14 & 3.3 & 0.1 & 27 & $40-1,39$ & 368.89 & 0.2 & 0.0 & 1 & Site 473 & & & & \\
\hline $83-1,44$ & 775.94 & 0.2 & 0.1 & 1 & $43-1,14$ & 387.14 & 7.7 & 0.3 & 62 & Site 473 & & & & \\
\hline $85-1,88$ & 795.38 & 8.7 & 0.7 & 67 & $43-1,131$ & 388.31 & 1.1 & 0.2 & 8 & $2-3,30$ & 3.80 & 2.0 & 1.6 & 3 \\
\hline $85-4,105$ & 800.05 & 2.0 & 0.6 & 11 & Site $\mathbf{4 7 0}$ & & & & & $4-2,30$ & 21.30 & 1.5 & 1.2 & 2 \\
\hline $87-4,49$ & 818.49 & 8.4 & 3.6 & 40 & Site $4 / 0$ & & & & & $6-2,20$ & 40.20 & 0.9 & 0.8 & 1 \\
\hline $89-1,55$ & 833.05 & 6.3 & 2.3 & 34 & $1-2,127$ & 2.77 & 0.5 & 0.4 & 1 & $8-2,119$ & 60.19 & 1.1 & 1.0 & 1 \\
\hline $91-5,50$ & 858.00 & 5.6 & 2.3 & 28 & $3-2,72$ & 21.22 & 0.4 & 0.3 & 1 & $10-1,23$ & 76.73 & 1.0 & 0.9 & 1 \\
\hline $92-1,105$ & 862.05 & - & 2.1 & - & $5-1,126$ & 39.26 & 3.3 & 0.2 & 26 & $12-1,104$ & 96.54 & 1.0 & 0.9 & 1 \\
\hline $94-1,54$ & 880.54 & - & 0.9 & - & $7-2,65$ & 59.15 & 1.0 & 0.3 & 6 & $14-1,20$ & 114.70 & 0.8 & 0.5 & 3 \\
\hline $96-1,67$ & 899.67 & 5.5 & 1.8 & 31 & $9-2,15$ & 77.65 & 1.6 & 0.4 & 10 & $16-5,10$ & 139.60 & 0.4 & 0.4 & 0 \\
\hline $97-1,44$ & 908.94 & 4.3 & 1.9 & 20 & $11-4,80$ & 100.30 & 0.8 & 0.3 & 4 & $18-2,120$ & 155.20 & 0.9 & 0.8 & 1 \\
\hline $99-1,50$ & 928.00 & 2.2 & 1.6 & 5 & $13-1,90$ & 114.90 & 0.3 & 0.3 & 0 & $20-3,20$ & 174.70 & 1.1 & 1.1 & 0 \\
\hline $101-1,27$ & 946.77 & 7.3 & 2.3 & 42 & $15-1,10$ & 133.10 & 6.0 & 0.3 & 48 & $23-2,9$ & 192.09 & 1.0 & 1.0 & 0 \\
\hline $104-2,76$ & 977.26 & 6.5 & 1.4 & 43 & $17-1,115$ & 153.15 & 6.3 & 0.2 & 51 & $25-2,22$ & 211.22 & 0.5 & 0.5 & 0 \\
\hline $107-2,0$ & 1005.00 & 4.2 & 3.8 & 3 & $18-2,65$ & 163.65 & 7.0 & 0.1 & 57 & $27-1,55$ & 229.05 & 0.2 & 0.2 & 0 \\
\hline $110-1,124$ & 1033.24 & 3.3 & 0.9 & 20 & & & & & & & & & & \\
\hline $110-3,5$ & 1035.05 & 4.3 & 1.9 & 21 & & & & & & & & & & \\
\hline
\end{tabular}

